Internat. J. Math. \& Math. Sci.

Vol. 24, No. 7 (2000) 437-440

S0161171200004129

(c) Hindawi Publishing Corp.

\title{
LEFT GLOBAL DIMENSIONS AND INVERSE POLYNOMIAL MODULES
}

\section{SANGWON PARK}

(Received 20 November 1999)

\begin{abstract}
We prove the fact l.gl. $\operatorname{dim} R[x]=(\lg g . \operatorname{dim} R)+1$, where l.gl.dim means the left global dimension by using inverse polynomial modules and injective dimensions. The classical way to prove the fact $\lg \operatorname{gl} \cdot \operatorname{dim} R[x]=(\lg \operatorname{gl} \cdot \operatorname{dim} R)+1$ is using polynomial modules and projective dimensions.
\end{abstract}

Keywords and phrases. Inverse polynomial module, injective module, short exact sequence, left global dimension.

2000 Mathematics Subject Classification. Primary 16E30, 13C11, 16D80.

1. Introduction. The classical way to prove the fact l.gl. $\operatorname{dim} R[x]=(\log . \operatorname{dim} R)+1$, where l.gl. dim means the left global dimension is using the construction $M[x]$ (where $M$ is any left $R$-module). In this paper, we give another proof of this fact by using inverse polynomial module $M\left[x^{-1}\right]$ and injective dimensions instead of polynomial module $M[x]$ and projective dimensions. Northcott [3] and McKerrow [1] showed that the polynomial module $M[x]$ and the inverse polynomial module $M\left[x^{-1}\right]$ are not isomorphic as left $R[x]$-modules by showing that if $R$ is a left noetherian ring and $E \neq 0$ is an injective left $R$-module, then $E\left[x^{-1}\right]$ is an injective left $R[x]$-module while $E[x]$ is not an injective left $R[x]$-module. Park in [5] also showed that if $P \neq 0$ is a projective left $R$-module, then $P\left[x^{-1}\right]$ is not a projective left $R[x]$-module while $P[x]$ is a projective left $R[x]$-module. Inverse polynomial modules were developed in $[1,3,4,5]$, and recently in [2].

Definition 1.1. Let $R$ be a ring and $M$ be a left $R$-module, then $M\left[x^{-1}\right]$ is a left $R[x]$-module such that

$$
x\left(m_{0}+m_{1} x^{-1}+\cdots+m_{n} x^{-n}\right)=m_{1}+m_{2} x^{-1}+\cdots+m_{n} x^{-n+1}
$$

and such that

$$
r\left(m_{0}+m_{1} x^{-1}+\cdots+m_{n} x^{-n}\right)=r m_{0}+r m_{1} x^{-1}+\cdots+r m_{n} x^{-n},
$$

where $r \in R$. Similarly, we can define $M\left[\left[x^{-1}\right]\right]$ as a left $R[x]$-module.

LEMMA 1.2. If $E\left[\left[x^{-1}\right]\right]$ is an injective left $R[x]$-module, then $E$ is an injective left $R$-module.

Proof. Let $I \subset R$ be a left ideal and let $f: I \rightarrow E$ be a $R$-linear map. Then the map $I\left[\left[x^{-1}\right]\right] \rightarrow E\left[\left[x^{-1}\right]\right]\left(\sum_{i=0}^{\infty} r_{i} x^{-i} \rightarrow \sum_{i=0}^{\infty} f\left(r_{i}\right) x^{-i}\right)$ is an $R[x]$-linear map so can be 
extended to a map

$$
R\left[\left[x^{-1}\right]\right] \stackrel{g}{\longrightarrow} E\left[\left[x^{-1}\right]\right] .
$$

Since $x R=0$ (for $R \subset R\left[\left[x^{-1}\right]\right]$ ), $x g(R)=0$ in $E\left[\left[x^{-1}\right]\right]$. But this implies $g(R) \subset E$ (with $E \subset E\left[\left[x^{-1}\right]\right]$ ). Then the map $R \rightarrow E$ agreeing with $g$ is an $R$-linear map and extends $I \rightarrow E$. So $E$ is an injective $R$-module.

THEOREM 1.3. Let $M$ be a left $R$-module, then

$$
\operatorname{inj} \operatorname{dim}_{R[x]}\left(M\left[\left[x^{-1}\right]\right]\right)=\operatorname{inj} \operatorname{dim}_{R}(M) .
$$

Proof. Let $R$ be a ring and $E$ be an injective left $R$-module. Define $\phi: \operatorname{Hom}_{R}(R[x], E)$ $\rightarrow E\left[\left[x^{-1}\right]\right]$ by $\phi(f)=f(1)+f(x) x^{-1}+f\left(x^{2}\right) x^{-2}+\cdots$, then $\operatorname{Hom}_{R}(R[x], E)$ and $E\left[\left[x^{-1}\right]\right]$ are isomorphic as left left $R[x]$-modules. Since $\operatorname{Hom}_{R}(R[x], E)$ is an injective left $R[x]$-module so is $E\left[\left[x^{-1}\right]\right]$. Suppose $\operatorname{inj} \operatorname{dim}_{R}(M)=n$ and

$$
0 \longrightarrow M \longrightarrow E^{0} \rightarrow E^{1} \longrightarrow \cdots \rightarrow E^{n} \longrightarrow 0
$$

is an injective resolution of $M$. Then

$$
0 \rightarrow M\left[\left[x^{-1}\right]\right] \rightarrow E^{0}\left[\left[x^{-1}\right]\right] \rightarrow E^{1}\left[\left[x^{-1}\right]\right] \rightarrow \cdots \rightarrow E^{n}\left[\left[x^{-1}\right]\right] \rightarrow 0
$$

is an injective resolution of $M\left[\left[x^{-1}\right]\right]$. Let

$$
K^{i}=\operatorname{ker}\left(E^{i} \longrightarrow E^{i+1}\right) \quad \text { for } 0 \leq i<n .
$$

Then $K^{i}$ is not an injective $R$-module for $0 \leq i<n$. So by the above lemma $K^{i}\left[\left[x^{-1}\right]\right]$ is not an injective $R[x]$-module. So then we get

$$
\operatorname{inj} \operatorname{dim}_{R[x]}\left(M\left[\left[x^{-1}\right]\right]\right)=n .
$$

Suppose $\operatorname{inj} \operatorname{dim}_{R}(M)=\infty$ and

$$
0 \rightarrow M \rightarrow E^{0} \rightarrow E^{1} \longrightarrow \cdots \rightarrow E^{n} \longrightarrow \cdots
$$

is an injective resolution of $M$. Then

$$
0 \rightarrow M\left[\left[x^{-1}\right]\right] \rightarrow E^{0}\left[\left[x^{-1}\right]\right] \rightarrow E^{1}\left[\left[x^{-1}\right]\right] \rightarrow \cdots \rightarrow E^{n}\left[\left[x^{-1}\right]\right] \rightarrow \cdots
$$

is an injective resolution of $M\left[\left[x^{-1}\right]\right]$. But $K^{i}$ is not an injective $R$-module for all $i$. Thus $K^{i}\left[\left[x^{-1}\right]\right]$ is not an injective $R[x]$-module for all $i$. Therefore, $\operatorname{inj} \operatorname{dim}_{R[x]}\left(M\left[\left[x^{-1}\right]\right]\right)=$ $\infty$. Similarly, if

$$
\operatorname{inj} \operatorname{dim}_{R[x]}\left(M\left[\left[x^{-1}\right]\right]\right)=n, \quad \text { then } \operatorname{inj} \operatorname{dim}_{R}(M)=n
$$

and if

$$
\operatorname{inj} \operatorname{dim}_{R[x]}\left(M\left[\left[x^{-1}\right]\right]\right)=\infty, \quad \text { then } \operatorname{inj} \operatorname{dim}_{R}(M)=\infty .
$$

Hence, $\operatorname{inj}_{\operatorname{dim}_{R[x]}}\left(M\left[\left[x^{-1}\right]\right]\right)=\operatorname{inj} \operatorname{dim}_{R}(M)$. 
THEOREM 1.4. Let $M, M\left[\left[X^{-1}\right]\right]$ be left $R[x]$-modules. Then there is a short exact sequence of $R[x]$-modules

$$
0 \rightarrow M \longrightarrow M\left[\left[x^{-1}\right]\right] \rightarrow M\left[\left[x^{-1}\right]\right] \longrightarrow 0 .
$$

Proof. Let $\phi: M \rightarrow\left[\left[x^{-1}\right]\right]$ be defined by

$$
\phi(y)=y+(x y) x^{-1}+\left(x^{2} y\right) x^{-2}+\left(x^{3} y\right) x^{-3}+\cdots, \quad \text { for } y \in M,
$$

then $\phi$ is an injective $R[x]$-linear map. Let $\psi: M\left[\left[x^{-1}\right]\right] \rightarrow M\left[\left[x^{-1}\right]\right]$ be defined by

$$
\psi\left(m_{0}+m_{1} x^{-1}+m_{2} x^{-2}+\cdots\right)=\left(m_{1}-x m_{0}\right)+\left(m_{2}-x m_{1}\right) x^{-1}+\cdots,
$$

then $\psi$ is a surjective $R[x]$-linear map. Let $y$ be an element of $M$, then

$$
\begin{aligned}
(\psi \circ \phi)(y) & =\psi\left(y+(x y) x^{-1}+\left(x^{2} y\right) x^{-2}+\cdots\right) \\
& =(x y-x y)+\left(x^{2} y-x^{2} y\right) x^{-1}+\left(x^{3} y-x^{3} y\right) x^{-2}+\cdots=0 .
\end{aligned}
$$

Therefore, image $(\phi) \subset \operatorname{ker}(\psi)$. Let $m_{0}+m_{1} x^{-1}+m_{2} x^{-2}+\cdots$ be an element of $\operatorname{ker}(\psi)$, then

$$
\begin{aligned}
\psi\left(m_{0}\right. & \left.+m_{1} x^{-1}+m_{2} x^{-2}+\cdots\right) \\
& =\left(m_{1}-x m_{0}\right)+\left(m_{2}-x m_{1}\right) x^{-1}+\left(m_{3}-x m_{2}\right) x^{-2}+\cdots=0 .
\end{aligned}
$$

Therefore, $m_{i+1}=x m_{i}$, for all $i=0,1,2, \ldots$. Then, for $m_{0} \in M$,

$$
\begin{aligned}
\phi\left(m_{0}\right) & =m_{0}+\left(x m_{0}\right) x^{-1}+\left(x^{2} m_{0}\right) x^{-2}+\cdots \\
& =m_{0}+m_{1} x^{-1}+m_{2} x^{-2}+\cdots, \quad \text { since } m_{i+1}=x m_{i} .
\end{aligned}
$$

So, $m_{0}+m_{1} x^{-1}+\cdots \in$ image $(\phi)$. Thus, $\operatorname{ker}(\psi) \subset$ image $(\phi)$. Therefore, image $(\phi)=$ $\operatorname{ker}(\psi)$. Hence, $0 \rightarrow M \rightarrow M\left[\left[x^{-1}\right]\right] \rightarrow M\left[\left[x^{-1}\right]\right] \rightarrow 0$ is a short exact sequence of $R[x]$ modules.

COROLLARY 1.5. $1 . g l . \operatorname{dim} R \leq 1 . g \mathrm{l} . \operatorname{dim} R[x] \leq \mathrm{l} \cdot \mathrm{gl} . \operatorname{dim} R+1$.

Proof. The first inequality follows from Theorem 1.3. The second follows from Theorems 1.4 and 1.3.

\section{Main theorem}

THEOREM 2.1. $\operatorname{l.gl} \cdot \operatorname{dim} R[x]=(\lg \operatorname{gl} \cdot \operatorname{dim} R)+1$.

Proof. From Corollary 1.5 we see that we only need argue that if $M \neq 0$ is a left $R$ module and if $\operatorname{inj} \operatorname{dim} M=n<\infty$, then there is a left $R[x]$-module $N$ with $\operatorname{inj} \operatorname{dim} N=$ $n+1$. In fact we show that $M$ itself can be made into such an $R[x]$-module. Let $M \neq 0$ be a left $R$-module and let $\operatorname{inj} \operatorname{dim} M=n<\infty$, and make $M$ into an $R[x]$-module with $x M=0$. By induction on $n$. Consider the short exact sequence of $R[x]$-modules

$$
0 \rightarrow M \rightarrow M\left[\left[x^{-1}\right]\right] \rightarrow M\left[\left[x^{-1}\right]\right] \longrightarrow 0 .
$$


From this short exact sequence we see that $\operatorname{inj}_{\operatorname{dim}}[x] M \leq n+1$, since $\operatorname{inj} \operatorname{dim}_{R[x]} M\left[\left[x^{-1}\right]\right]=\operatorname{inj} \operatorname{dim}_{R} M=n$. So we only need to prove that for any $n$, if $\operatorname{inj} \operatorname{dim}_{R} M=n$, then inj $\operatorname{dim}_{R[x]} M>n$. If $n=0$, then $M$ is an injective left $R$-module. But any injective $R[x]$-module is $x$-divisible. Also $x M=0$. So $M$ is not $x$-divisible. Hence, $M$ is not an injective $R[x]$-module. Now suppose $n=1$. Then we have an exact sequence

$$
0 \longrightarrow M \longrightarrow E \longrightarrow \frac{E}{M} \longrightarrow 0
$$

with $E$ and $E / M$ injective left $R$-modules. Since $M$ is a submodule of $E\left[\left[x^{-1}\right]\right]$ which is an injective left $R[x]$-module as left $R[x]$-module, we have the short exact sequence of $R[x]$-modules

$$
0 \rightarrow M \rightarrow E\left[\left[x^{-1}\right]\right] \rightarrow \frac{E\left[\left[x^{-1}\right]\right]}{M} \rightarrow 0
$$

Now we want to argue $E\left[\left[x^{-1}\right]\right] / M$ is not an injective left $R[x]$-module. Suppose $E\left[\left[x^{-1}\right]\right] / M$ is an injective left $R[x]$-module. Let $I=(x) \subset R[x]$. Consider the submodule of all $z$ in $E\left[\left[x^{-1}\right]\right] / M$ such that $x z=0$. Then this submodule of $E\left[\left[x^{-1}\right]\right] / M$ is isomorphic to $E / M \oplus M x^{-1}$ as an $R[x] /(x) \cong R$ module, i.e., is isomorphic to $E / M \oplus M$. So if $E\left[\left[x^{-1}\right]\right] / M$ were an injective left $R[x]$-module, then $E / M \oplus M$, and so $M$, would be an injective left $R$-module. Therefore by this contradiction we see that $E\left[\left[x^{-1}\right]\right] / M$ is not an injective left $R[x]$-module. Now we suppose inj $\operatorname{dim} M=n>1$ and make the induction hypothesis. Let

$$
0 \rightarrow M \longrightarrow E \rightarrow C \longrightarrow 0
$$

be an exact sequence of left $R$-modules with $E$ an injective left $R$-module. Then $\operatorname{inj} \operatorname{dim} C=n-1$. Make this into an exact sequence of left $R[x]$-modules with $x M=$ $0, x E=0$, and $x C=0$. Then by the induction hypothesis $\operatorname{inj}_{\operatorname{dim}} \operatorname{dix}_{R[} E=1$, $\operatorname{inj} \operatorname{dim}_{R[x]} C=(n-1)+1=n$. Then since $n>1$ we get that $\operatorname{inj}_{\operatorname{dim}} \operatorname{dix}_{R} M=n+1$. This implies l.gl. $\operatorname{dim} R[x]=(\operatorname{l.gl} . \operatorname{dim} R)+1$.

\section{REFERENCES}

[1] A. S. McKerrow, On the injective dimension of modules of power series, Quart. J. Math. Oxford Ser. (2) 25 (1974), 359-368. MR 51\#8098. Zbl 302.16027.

[2] L. Melkersson, Content and inverse polynomials on Artinian modules, Comm. Algebra 26 (1998), no. 4, 1141-1145. MR 98k:16030. Zbl 931.13005.

[3] D. G. Northcott, Injective envelopes and inverse polynomials, J. London Math. Soc. (2) 8 (1974), 290-296. MR 50\#13003. Zbl 284.13012.

[4] S. Park, Inverse polynomials and injective covers, Comm. Algebra 21 (1993), no. 12, 45994613. MR 94k:16008. Zbl 794.16004.

[5] _ The Macaulay-Northcott functor, Arch. Math. (Basel) 63 (1994), no. 3, 225-230. MR 95f:16004. Zbl 804.18009.

Sangwon Park: Department of Mathematics, Dong-A University, Pusan, 604-714, KOREA

E-mail address: swpark@mai 1.donga.ac.kr 


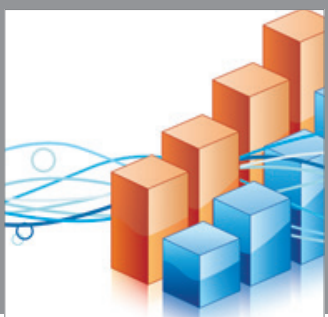

Advances in

Operations Research

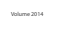

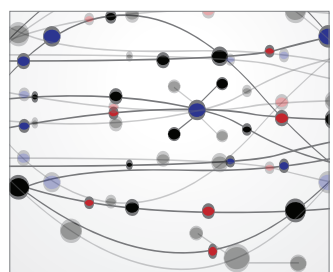

\section{The Scientific} World Journal
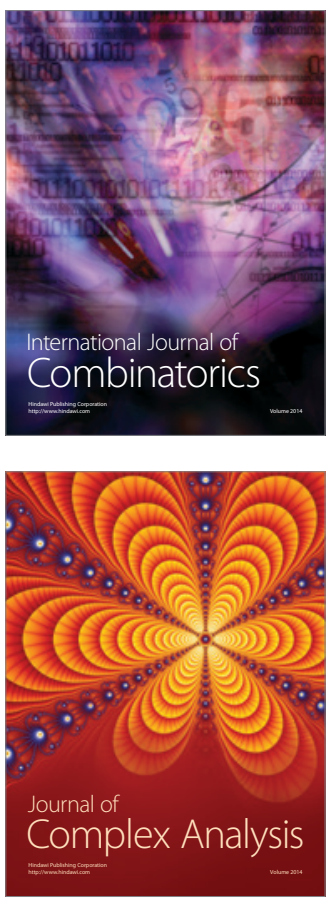

International Journal of

Mathematics and

Mathematical

Sciences
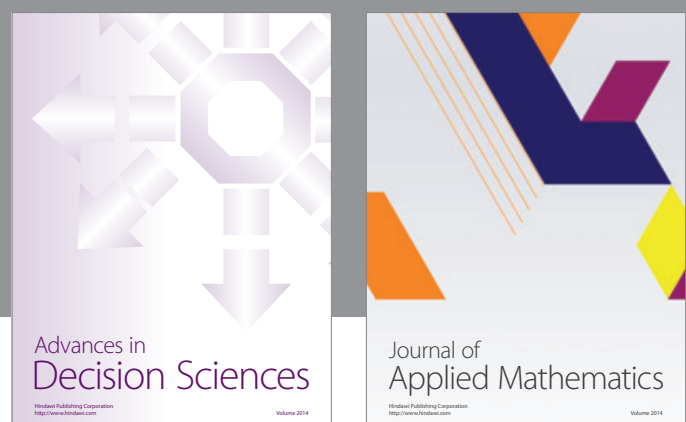

Journal of

Applied Mathematics
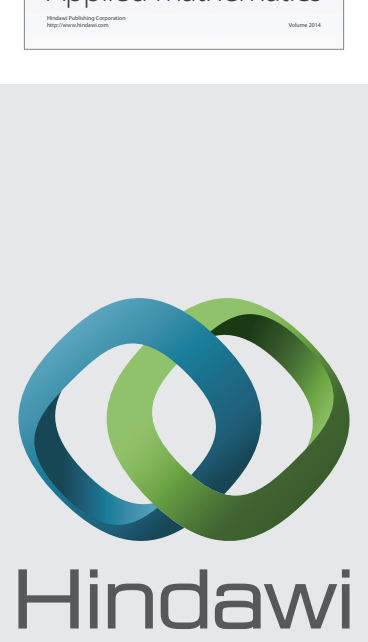

Submit your manuscripts at http://www.hindawi.com
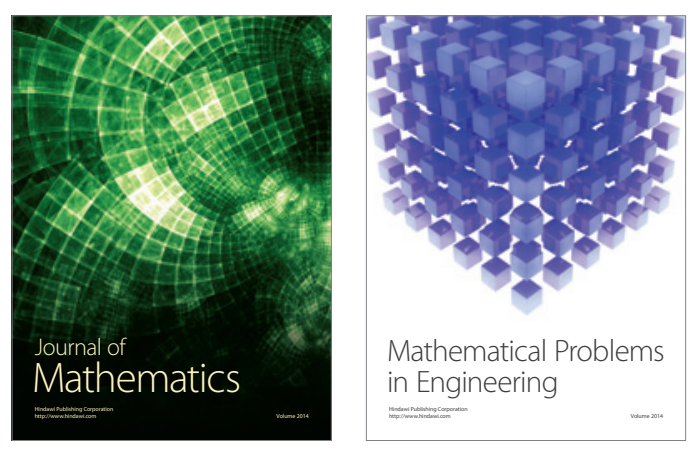

Mathematical Problems in Engineering
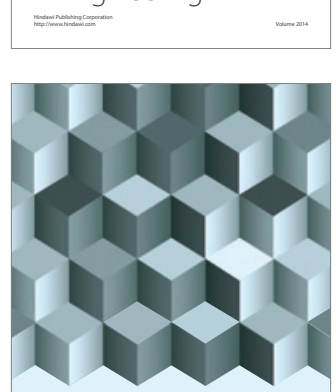

Journal of

Function Spaces
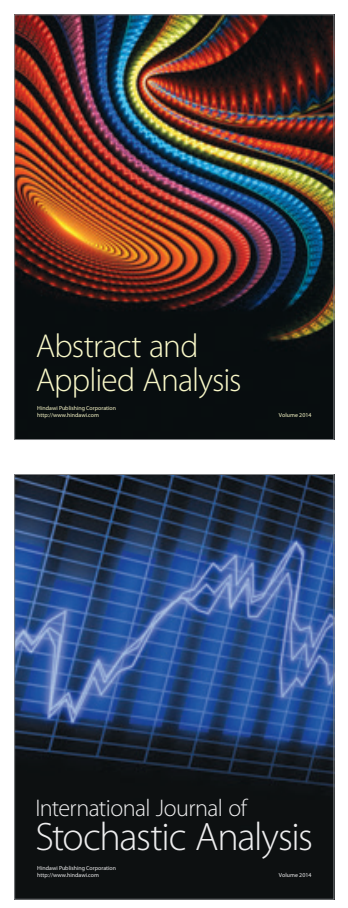

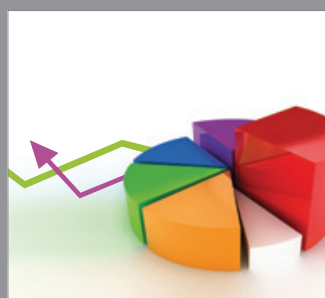

ournal of

Probability and Statistics

Promensencen
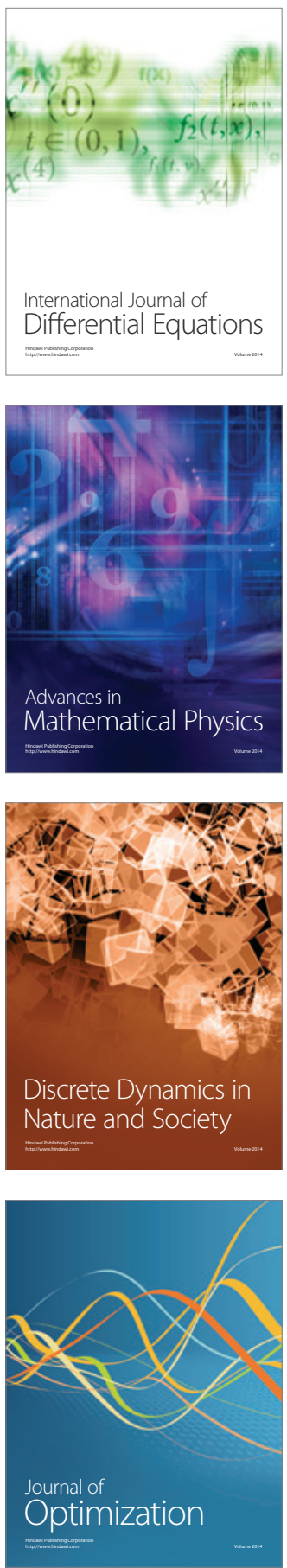